\title{
Derivations and automorphisms of twisted deformative Schrödinger-Virasoro Lie algebras
}

\author{
Wei Wang ${ }^{1,2)}$, Junbo $\mathrm{Li}^{2,3)}$, Ying $\mathrm{Xu}^{2)}$ \\ ${ }^{1)}$ School of Mathematics and Computer Science, Ningxia University, Yinchuan 750021, China \\ ${ }^{2)}$ Wu Wen-Tsun Key Laboratory of Mathematics, USTC, Hefei 230026, China \\ ${ }^{3)}$ School of Mathematics and Statistics, Changshu Institute of Technology, Changshu 215500, China
}

Abstract: In this paper the derivation algebra and automorphism group of the twisted deformative Schrödinger-Virasoro Lie algebras are determined.

Key words: Schrödinger-Virasoro Lie algebras, derivations, automorphisms.

Mathematics Subject Classification (2010): 17B05, 17B40, 17B65, $17 \mathrm{~B} 68$.

\section{Introduction}

It is well known that the Virasoro algebra plays an important role in many areas of theoretical physics and mathematics, which occures in the investigation of conformal field theory and has a $\mathbb{C}$-basis $\left\{L_{n}, c \mid n \in \mathbb{Z}\right\}$ with the nontrivial relations $\left[L_{n}, L_{m}\right]=(m-n) L_{n+m}$. It can be regarded as the complexification of the Lie algebra of polynomial vector fields on a circle, and also as the Lie algebra of derivations of the ring $\mathbb{C}\left[z, z^{-1}\right]$. The centerless Virasoro algebra admits many kinds of extensions, one of these is the Schrödinger-Virasoro type Lie algebras (see [7-9]), firstly introduced in [2] in the context of non-equilibrium statistical physics during the process of investigating the free Schrödinger equations and closely related to the Schrödinger algebra and the Virasoro algebra. Recently the vertex algebra representations, Lie bialgebra structures, irreducible weight modules with finitedimensional weight spaces and also Wittaker modules of the Schrödinger-Witt Lie algebras were extensively investigated in [10 12, 16]. The generalization of the Schrödinger-Virasoro Lie algebras was introduced in [15], whose automorphism groups and Verma modules were described therein.

For any $\lambda, \mu \in \mathbb{C},[9]$ introduced a family of infinite-dimensional Lie algebras called twisted deformative Schrödinger-Virasoro Lie algebras, admitting $\mathbb{C}$-basis $\left\{L_{n}, Y_{n}, M_{n} \mid n \in \mathbb{Z}\right\}$ and the following Lie brackets

$$
\begin{array}{ll}
{\left[L_{n}, L_{m}\right]=(m-n) L_{n+m},} & \\
{\left[L_{n}, Y_{m}\right]=\left(m-\frac{\lambda+1}{2} n+\mu\right) Y_{n+m},} & {\left[Y_{n}, Y_{m}\right]=(m-n) M_{n+m},} \\
{\left[L_{n}, M_{m}\right]=(m-\lambda n+2 \mu) M_{n+m}, \quad\left[Y_{n}, M_{m}\right]=\left[M_{n}, M_{m}\right]=0 .}
\end{array}
$$

Supported by NSF grants 10825101, 10861004 of China, China Postdoctoral Science Foundation grant 201003326 and the Natural Science Research Projects of Jiangsu Education Committee grant 09KJB110001

Electronic mail: wwll@mail.ustc.edu.cn, sd_junbo@163.com, xying@mail.ustc.edu.cn 
We denote this Lie algebra by $\mathscr{L}$, which is $\mathbb{Z}$-graded with

$$
\mathscr{L}=\bigoplus_{n \in \mathbb{Z}} \mathbb{C} \mathscr{L}_{n}, \quad \mathscr{L}_{n}=\mathbb{C} L_{n} \oplus \mathbb{C} Y_{n} \oplus \mathbb{C} M_{n}, \quad\left[\mathscr{L}_{n}, \mathscr{L}_{m}\right] \subseteq \mathscr{L}_{n+m}
$$

For convenience we introduce the following notations

$$
\mathcal{L}=\sum_{n \in \mathbb{Z}} \mathbb{C} L_{n}, \quad \mathcal{Y}=\sum_{n \in \mathbb{Z}} \mathbb{C} Y_{n}, \quad \mathcal{M}=\sum_{n \in \mathbb{Z}} \mathbb{C} M_{n}
$$

Then $\mathcal{L}$ is the centerless Virasoro algebra, $\mathcal{I}=\mathcal{Y} \oplus \mathcal{M}$ is the unique maximal ideal of $\mathscr{L}$ and $\mathcal{M}$ is the center of $\mathcal{I}$.

It is well known that the determination of derivations and automorphisms plays an important part in the investigation of the structure and representation of the relevant Lie algebras. Many references (see [1, 3-6, 9, 17, 18]) have focused on derivations and automorphisms of different Lie algebra backgrounds.

Note that if $\mu \in \mathbb{Z}$, then $\left\{L_{n}, Y_{n-\mu}, M_{n-2 \mu}\right\}$ is a basis of $\mathscr{L}$. Hence one can assume $\mu=0$ if $\mu \in \mathbb{Z}$. Especially, for the case $\lambda=\mu=0, \mathscr{L}$ is nothing but the twisted Schrödinger-Virasoro algebra, whose derivations and automorphisms were determined in [13]. If $\mu \in \frac{1}{2}+\mathbb{Z}$, one can assume $\mu=\frac{1}{2}$ by shifting basis, in which case the derivations were investigated in [14] and automorphisms for the special case $\lambda=0$ were determined in [15]. Furthermore, for the case $\mu=\frac{1}{2}$ and $\lambda \neq 0$, one can obtain the corresponding results on automorphisms by following the proof of Theorem 3.2 in [15]. Thus, in this paper we always make the following assumptions on $\mu$ and $\lambda$

$$
\mu \notin \frac{1}{2}+\mathbb{Z}, \quad \mu=0 \text { and } \lambda \neq 0 \text { if } \mu \in \mathbb{Z} .
$$

In the following two sections, we shall determine the derivation algebra (see Theorem 2.5) and the automorphism group (see Theorem 3.4) of $\mathscr{L}$ under the assumptions made in (1.1).

\section{Derivation algebra of $\mathscr{L}$}

A linear map $d: \mathscr{L} \rightarrow \mathscr{L}$ is called a derivation of $\mathscr{L}$, if $d([x, y])=[d(x), y]+[x, d(y)]$ holds for any $x, y \in \mathscr{L}$. For any fixed $z \in \mathscr{L}$, the linear map $\operatorname{ad}_{z}: \mathscr{L} \rightarrow \mathscr{L}$ is called an inner derivation if $\operatorname{ad}_{z}(x)=[z, x]$ for any $x \in \mathscr{L}$. Denote by $\operatorname{Der} \mathscr{L}$ and ad $\mathscr{L}$ respectively the vector spaces of all derivations and inner derivations. Then the first homological group $\mathcal{H}^{1}(\mathscr{L}, \mathscr{L}) \cong \operatorname{Der} \mathscr{L} / \operatorname{ad} \mathscr{L}$

Firstly, we give the following description of $\operatorname{Der} \mathscr{L}$.

Lemma 2.1 $\operatorname{Der} \mathscr{L}=(\operatorname{Der} \mathscr{L})_{0}+$ ad $\mathscr{L}$, where

$$
(\operatorname{Der} \mathscr{L})_{0}=\left\{d \in \operatorname{Der} \mathscr{L} \mid d\left(\mathscr{L}_{n}\right) \subseteq \mathscr{L}_{n}, \forall n \in \mathbb{Z}\right\}
$$


Proof By Proposition 1.2 of [1], we need two steps to complete the proof of this lemma.

Step 1 For any $n \neq 0$, if $d \in \mathcal{H}^{1}\left(\mathscr{L}_{0}, \mathscr{L}_{n}\right)$, then $d \in$ ad $\mathscr{L}$.

Since $\mathscr{L}_{n}=\mathbb{C} L_{n} \oplus \mathbb{C} Y_{n} \oplus \mathbb{C} M_{n}$ for all $n \in \mathbb{Z}$, one can assume $d\left(X_{0}\right)=e_{1}^{X} L_{n}+e_{2}^{X} Y_{n}+e_{3}^{X} M_{n}$ for some $e_{i}^{X} \in \mathbb{C}, i=1,2,3, X \in\{L, Y, M\}$. Applying $d$ to $\left[L_{0}, Y_{0}\right]=\mu Y_{0},\left[L_{0}, M_{0}\right]=2 \mu M_{0}$ and $\left[Y_{0}, M_{0}\right]=0$ respectively, we obtain

$$
\left\{\begin{array}{l}
(n-\mu) e_{1}^{Y}=\left(\mu-\frac{1+\lambda}{2} n\right) e_{1}^{L}+n e_{2}^{Y}=n e_{2}^{L}-(n+\mu) e_{3}^{Y}=0 \\
(n-2 \mu) e_{1}^{M}=(n-\mu) e_{2}^{M}=(2 \mu-\lambda n) e_{1}^{L}-n e_{3}^{M}=0 \\
(2 \mu-\lambda n) e_{1}^{Y}+n e_{2}^{M}=\left(\mu-\frac{1+\lambda}{2} n\right) e_{1}^{M}=0
\end{array}\right.
$$

which combined with (1.1) give

$$
e_{1}^{Y}=e_{1}^{M}=e_{2}^{M}=0, \quad e_{2}^{Y}=\frac{\lambda-2 \mu+1}{2 n} e_{1}^{L}, \quad e_{3}^{Y}=\frac{n}{n+\mu} e_{2}^{L}, \quad e_{3}^{M}=\frac{2 \mu-\lambda n}{n} e_{1}^{L} .
$$

Hence, denoting $\alpha=\frac{1}{n} e_{1}^{L} L_{n}+\frac{1}{n+\mu} e_{2}^{L} Y_{n}+\frac{1}{n+2 \mu} e_{3}^{L} M_{n}$, we obtain $d\left(X_{0}\right)=\left[X_{0}, \alpha\right]$ for any $X \in\{L, Y, M\}$.

Step 2 For any $n \neq m$, if $f \in \operatorname{Hom}_{\mathscr{L}_{0}}\left(\mathscr{L}_{n}, \mathscr{L}_{m}\right)$, then $f=0$.

Assume that $f\left(X_{n}\right)=c_{1}^{X} L_{m}+c_{2}^{X} Y_{m}+c_{3}^{X} M_{m}$ for some $c_{i}^{X} \in \mathbb{C}$ with $X \in\{L, Y, M\}, i=1,2,3$. Applying $f$ to both sides of the following three identities

$$
\left[L_{0}, L_{n}\right]=n L_{n},\left[L_{0}, Y_{n}\right]=(n+\mu) Y_{n},\left[L_{0}, M_{n}\right]=(n+2 \mu) M_{n}
$$

and comparing the coefficients of $L_{m}, Y_{m}$ and $M_{m}$ respectively, we have

$$
\left\{\begin{array}{l}
(m-n) c_{1}^{L}=(m-n+\mu) c_{2}^{L}=(m-n+2 \mu) c_{3}^{L}=0, \\
(m-n-\mu) c_{1}^{Y}=(m-n) c_{2}^{Y}=(m-n+\mu) c_{3}^{Y}=0, \\
(m-n-2 \mu) c_{1}^{M}=(m-n-\mu) c_{2}^{M}=(n+2 \mu) c_{3}^{M}=0 .
\end{array}\right.
$$

Note that $\mu$ satisfies (1.1), one can deduce $c_{i}^{X}=0, X \in\{L, Y, M\}, i=1,2,3$. Thus $f=0$.

Let $d \in(\operatorname{Der} \mathscr{L})_{0}$. For $n \in \mathbb{Z}$ and $X \in\{L, Y, M\}$, one can assume

$$
d\left(X_{n}\right)=f_{1}^{X}(n) L_{n}+f_{2}^{X}(n) Y_{n}+f_{3}^{X}(n) M_{n} \quad \text { for some } f_{i}^{X}(n) \in \mathbb{C}, i=1,2,3 \text {. }
$$

Lemma 2.2 For any $n \in \mathbb{Z}$ and some $a, b, c, \bar{c}, e, \bar{e} \in \mathbb{C}$, one can assume

(1) $f_{1}^{L}(n)=a n$.

(2) $f_{2}^{L}(n)= \begin{cases}\frac{e}{2 \mu}(2 \mu-(\lambda+1) n) & \text { if } \mu \notin \mathbb{Z}, \\ b n\left(1-\delta_{\lambda,-1}\right) & \text { if } \mu=0 .\end{cases}$ 
(3) If $\mu \notin \mathbb{Z}$, then $f_{3}^{L}(n)=\frac{\bar{e}}{2 \mu}(2 \mu-\lambda n)$.

(4) If $\mu=0$, then

$$
f_{3}^{L}(n)= \begin{cases}\frac{c}{6}\left(n^{3}-n\right)-\frac{\bar{c}}{3}\left(n^{3}-4 n\right) & \text { if } \lambda=-2, \\ \frac{c}{2}\left(n^{2}-n\right)-\bar{c}\left(n^{2}-2 n\right) & \text { if } \lambda=-1, \\ \bar{c} n & \text { if } \lambda \notin\{-2,0,-1\} .\end{cases}
$$

Proof (1) Applying $d$ to $\left[L_{n}, L_{m}\right]=(m-n) L_{m+n}$ and comparing the coefficients of $L_{m+n}$, we have $(m-n)\left(f_{1}^{L}(m+n)-f_{1}^{L}(m)-f_{1}^{L}(n)\right)=0$, which gives

$$
f_{1}^{L}(m+n)=f_{1}^{L}(m)+f_{1}^{L}(n) \text { if } m \neq n .
$$

Taking $n=0$ in (2.1), we have $f_{1}^{L}(0)=0$. Applying (2.1), one can deduce

$$
f_{1}^{L}(2 n)=f_{1}^{L}(3 n)+f_{1}^{L}(-n)=f_{1}^{L}(2 n)+f_{1}^{L}(n)+f_{1}^{L}(-2 n)+f_{1}^{L}(n)=2 f_{1}^{L}(n),
$$

which together with (2.1) gives $f_{1}^{L}(m+n)=f_{1}^{L}(m)+f_{1}^{L}(n)$ for all $m, n \in \mathbb{Z}$. Thus $f_{1}^{L}(n)=f_{1}^{L}(n-1)+f_{1}^{L}(1)$. Hence, by induction on $n$, one can deduce $f_{1}^{L}(n)=n f_{1}^{L}(1)$ for all $n \in \mathbb{Z}$.

(2) Applying $d$ to $\left[L_{n}, L_{m}\right]=(m-n) L_{m+n}$ and comparing the coefficients of $Y_{m+n}$, one has

$$
(2 m-(\lambda+1) n+2 \mu) f_{2}^{L}(m)-(2 n-(\lambda+1) m+2 \mu) f_{2}^{L}(n)=2(m-n) f_{2}^{L}(m+n) .
$$

Case $1 \mu \notin \mathbb{Z}$.

Setting $n=0$ in (2.2), we obtain $2 \mu f_{2}^{L}(m)=(2 \mu-(\lambda+1) m) f_{2}^{L}(0)$, which gives $f_{2}^{L}(m)=$ $\frac{1}{2 \mu}(2 \mu-(\lambda+1) m) f_{2}^{L}(0)$ for all $m \in \mathbb{Z}$.

Case $2 \mu=0$.

Taking $\mu=0$ in (2.2), we have

$$
(2 m-(\lambda+1) n) f_{2}^{L}(m)-(2 n-(\lambda+1) m) f_{2}^{L}(n)=2(m-n) f_{2}^{L}(m+n) .
$$

Taking $n=1,2$ in (2.3), we obtain

$$
\begin{aligned}
& 2(m-1) f_{2}^{L}(m+1)=(2 m-\lambda-1) f_{2}^{L}(m)-(2-(\lambda+1) m) f_{2}^{L}(1), \\
& 2(m-2) f_{2}^{L}(m+2)=2(m-\lambda-1) f_{2}^{L}(m)-(4-(\lambda+1) m) f_{2}^{L}(2) .
\end{aligned}
$$

Taking $n=1$ and replacing $m$ by $m+1$ in (2.3), one can deduce

$$
(2 m-\lambda+1) f_{2}^{L}(m+1)-(2-(\lambda+1)(m+1)) f_{2}^{L}(1)=2 m f_{2}^{L}(m+2) .
$$


Multiplying (2.6) by $2(m-1)(m-2)$, then using (2.4) and (2.5), we obtain

$$
\begin{aligned}
& (\lambda-1)(2 \lambda-(5+\lambda) m+2) f_{2}^{L}(m) \\
= & m(m-2)\left(4(1+\lambda) m-\lambda^{2}-7\right) f_{2}^{L}(1)+2 m(m-1)(4-(\lambda+1) m) f_{2}^{L}(2) .
\end{aligned}
$$

Applying $d$ to $\left[L_{n}, Y_{m}\right]=\left(m-\frac{\lambda+1}{2} n\right) Y_{m+n}$ and comparing the coefficients of $M_{m+n}$, we have

$$
2(m-n) f_{2}^{L}(n)+2(m-\lambda n) f_{3}^{Y}(m)-(2 m-(1+\lambda) n) f_{3}^{Y}(n+m)=0 .
$$

Setting $m=0$ in (2.8), we obtain

$$
(1+\lambda) n f_{3}^{Y}(n)=2 n f_{2}^{L}(n)+2 \lambda n f_{3}^{Y}(0) .
$$

Replacing $n$ by $-n, m$ by $n$ in (2.8), one has

$$
(\lambda+3) n f_{3}^{Y}(0)=2(1+\lambda) n f_{3}^{Y}(n)+4 n f_{2}^{L}(-n) .
$$

Multiplying (2.9) by $(\lambda+3)$ and using (2.10), we obtain

$$
3\left(1-\lambda^{2}\right) n f_{3}^{Y}(n)=2(\lambda+3) n f_{2}^{L}(n)+8 \lambda n f_{2}^{L}(-n) .
$$

Taking $n=1,2$ in (2.11) respectively, one has

$$
\begin{aligned}
& 3\left(1-\lambda^{2}\right) f_{3}^{Y}(1)=2(\lambda+3) f_{2}^{L}(1)+8 \lambda f_{2}^{L}(-1), \\
& 3\left(1-\lambda^{2}\right) f_{3}^{Y}(2)=2(\lambda+3) f_{2}^{L}(2)+8 \lambda f_{2}^{L}(-2) .
\end{aligned}
$$

Furthermore, setting $n=m=1$ in (2.8), we obtain

$$
(1-\lambda)\left(f_{3}^{Y}(2)-2 f_{3}^{Y}(1)\right)=0
$$

Multiplying (2.14) by $3(1+\lambda)$, using (2.12) and (2.13), one can deduce

$$
(\lambda+3)\left(f_{2}^{L}(2)-2 f_{2}^{L}(1)\right)+4 \lambda\left(f_{2}^{L}(-2)-2 f_{2}^{L}(-1)\right)=0 .
$$

Subcase $1 \lambda=-5$.

Taking $\lambda=-5$ in (2.7), one has

$$
f_{2}^{L}(m)=\frac{1}{6} m(m-1)(m+1) f_{2}^{L}(2)-\frac{1}{3} m(m-2)(m+2) f_{2}^{L}(1) .
$$

Taking $m=-1,-2$ in (2.16), we obtain $f_{2}^{L}(-1)=-f_{2}^{L}(1)$ and $f_{2}^{L}(-2)=-f_{2}^{L}(2)$. Then (2.15) gives $f_{2}^{L}(2)=2 f_{2}^{L}(1)$, which together with (2.16), admits $f_{2}^{L}(m)=m f_{2}^{L}(1)$ for all $m \in \mathbb{Z}$. 
Subcase $2 \lambda=-3$.

Taking $\lambda=-3$ in (2.7), one has

$$
2(m+2) f_{2}^{L}(m)=m(m+2)(m-1) f_{2}^{L}(2)-2 m(m+2)(m-2) f_{2}^{L}(1),
$$

which gives

$$
f_{2}^{L}(m)=\frac{1}{2} m(m-1) f_{2}^{L}(2)-m(m-2) f_{2}^{L}(1) \text { if } m \neq-2 .
$$

Setting $\lambda=-3, m=1$ and $n=-2$ in (2.3), we have

$$
f_{2}^{L}(-2)=f_{2}^{L}(1)+3 f_{2}^{L}(-1)
$$

Furthermore, taking $m=-1$ in (2.18), one has

$$
f_{2}^{L}(-1)=f_{2}^{L}(2)-3 f_{2}^{L}(1)
$$

Applying this by substituting for the second term of the right-hand side in (2.19), we have

$$
f_{2}^{L}(-2)=3 f_{2}^{L}(2)-8 f_{2}^{L}(1)
$$

Thus (2.18) holds for all $m \in \mathbb{Z}$. Taking $\lambda=-3$ in (2.15), then using (2.20) and (2.21), one can deduce $f_{2}^{L}(2)=2 f_{2}^{L}(1)$. Thus (2.18) gives $f_{2}^{L}(m)=m f_{2}^{L}(1)$ for all $m \in \mathbb{Z}$.

Subcase $3 \lambda=-1$.

Letting $\lambda=-1$ in (2.7) gives

$$
f_{2}^{L}(m)=(m-1) f_{2}^{L}(2)-(m-2) f_{2}^{L}(1) \text { if } m \neq 0
$$

Taking $m=-1$ in (2.22), we have

$$
f_{2}^{L}(-1)=-2 f_{2}^{L}(2)+3 f_{2}^{L}(1)
$$

Furthermore, letting $\lambda=-1, m=1, n=-1$ in (2.3), we have $f_{2}^{L}(1)+f_{2}^{L}(-1)=2 f_{2}^{L}(0)$. Using this in (2.23), one has $f_{2}^{L}(0)=-f_{2}^{L}(2)+2 f_{2}^{L}(1)$. Thus (2.22) holds for all $m \in \mathbb{Z}$. Taking $m=-2$ in (2.22), we obtain

$$
f_{2}^{L}(-2)=-3 f_{2}^{L}(2)+4 f_{2}^{L}(1)
$$

Taking $\lambda=-1$ in (2.15), then using (2.23) and (2.24), one can deduce $f_{2}^{L}(2)=2 f_{2}^{L}(1)$. Thus (2.22) gives $f_{2}^{L}(m)=m f_{2}^{L}(1)$ for all $m \in \mathbb{Z}$. Taking $\lambda=-1$ in (2.12) and using $f_{2}^{L}(-1)=-f_{2}^{L}(1)$, one can deduce $f_{2}^{L}(1)=0$. Thus $f_{2}^{L}(n)=0$ for all $n \in \mathbb{Z}$. 
Subcase $4 \lambda=1$.

Letting $\lambda=1$ in (2.3), we obtain $(m-n)\left(f_{2}^{L}(m+n)-f_{2}^{L}(n)-f_{2}^{L}(m)\right)=0$. Utilizing the similar technique to that of $(1)$, one can deduce $f_{2}^{L}(m)=m f_{2}^{L}(1)$ for all $m \in \mathbb{Z}$.

Subcase $5 \lambda \notin\{-5,-3, \pm 1\}$.

Taking $n=0$ in (2.3), we have $(\lambda+1) m f_{2}^{L}(0)=0$ for all $m \in \mathbb{Z}$. This forces $f_{2}^{L}(0)=0$. Using this and replacing $n$ by $-m$ in (2.3) , we obtain $(\lambda+3)\left(f_{2}^{L}(m)+f_{2}^{L}(-m)\right)=0$. Thus $f_{2}^{L}(m)=-f_{2}^{L}(-m)$ for all $m \in \mathbb{Z}$ since $\lambda \neq-3$. Thus

$$
f_{2}^{L}(1)=-f_{2}^{L}(-1), \quad f_{2}^{L}(2)=-f_{2}^{L}(-2) .
$$

Taking $n=2$ in (2.3), we have

$$
2(m-\lambda-1) f_{2}^{L}(m)-(4-(\lambda+1) m) f_{2}^{L}(2)=2(m-2) f_{2}^{L}(m+2) .
$$

Replacing $m$ by $m+2, n$ by -2 in (2.3) and using (2.25), we have

$$
2(m+\lambda+3) f_{2}^{L}(m+2)-(4+(\lambda+1)(m+2)) f_{2}^{L}(2)=2(m+4) f_{2}^{L}(m) .
$$

Using (2.26) and (2.27), one can deduce $(\lambda+5)(\lambda-1)\left(2 f_{2}^{L}(m)-m f_{2}^{L}(2)\right)=0$, which gives $f_{2}^{L}(m)=\frac{1}{2} f_{2}^{L}(2) m$. Taking $m=2, n=-1$ in (2.3) and using (2.25), we can deduce $f_{2}^{L}(2)=2 f_{2}^{L}(1)$. Thus $f_{2}^{L}(m)=m f_{2}^{L}(1)$ for all $m \in \mathbb{Z}$. We have completed the proof of $(2)$.

Next we begin the proof of (3) and (4) of this lemma. Applying $d$ to $\left[L_{n}, L_{m}\right]=(m-$ n) $L_{m+n}$, then comparing the coefficients of $M_{m+n}$, one has

$$
(m-\lambda n+2 \mu) f_{3}^{L}(m)-(n-\lambda m+2 \mu) f_{3}^{L}(n)=(m-n) f_{3}^{L}(m+n) .
$$

(3) If $\mu \notin \mathbb{Z}$, then taking $n=0$ in (2.28), we have $2 \mu f_{3}^{L}(m)=(2 \mu-\lambda m) f_{3}^{L}(0)$, which gives

$$
f_{3}^{L}(m)=\frac{1}{2 \mu}(2 \mu-\lambda m) f_{3}^{L}(0) .
$$

(4) Taking $\mu=0$ in (2.28), we have

$$
(m-\lambda n) f_{3}^{L}(m)-(n-\lambda m) f_{3}^{L}(n)=(m-n) f_{3}^{L}(m+n) .
$$

Taking $n=1,2$ in (2.29), we have

$$
\begin{aligned}
& (m-\lambda) f_{3}^{L}(m)-(1-\lambda m) f_{3}^{L}(1)=(m-1) f_{3}^{L}(m+1), \\
& (m-2 \lambda) f_{3}^{L}(m)-(2-\lambda m) f_{3}^{L}(2)=(m-2) f_{3}^{L}(m+2) .
\end{aligned}
$$


Setting $n=1$ and replacing $m$ by $m+1$ in (2.29), one can deduce

$$
(m+1-\lambda) f_{3}^{L}(m+1)-(1-\lambda(m+1)) f_{3}^{L}(1)=m f_{3}^{L}(m+2) .
$$

Thus the three equations (2.30)-(2.32) imply

$$
\begin{aligned}
& (\lambda-1)((\lambda+2) m-2 \lambda) f_{3}^{L}(m) \\
= & m(m-2)\left(2-\lambda+\lambda^{2}-2 \lambda m\right) f_{3}^{L}(1)-m(m-1)(2-\lambda m) f_{3}^{L}(2) .
\end{aligned}
$$

Case $1 \lambda=-2$.

Setting $\lambda=-2$ in (2.33), one has

$$
f_{3}^{L}(m)=\frac{1}{6} m(m+1)(m-1) f_{3}^{L}(2)-\frac{1}{3} m(m-2)(m+2) f_{3}^{L}(1), \quad \forall m \in \mathbb{Z} .
$$

Case $2 \lambda=-1$.

In this case (2.33) gives

$$
f_{3}^{L}(m)=\frac{1}{2} m(m-1) f_{3}^{L}(2)-m(m-2) f_{3}^{L}(1) \text { for } m \neq-2 .
$$

Taking $\lambda=-1, m=-2$ and $n=1$ in (2.29), one has

$$
f_{3}^{L}(-2)=3 f_{3}^{L}(-1)+f_{3}^{L}(1)
$$

Setting $m=-1$ in (2.34), we have $f_{3}^{L}(-1)=f_{3}^{L}(2)-3 f_{3}^{L}(1)$. Using this in (2.35), we obtain $f_{3}^{L}(-2)=3 f_{3}^{L}(2)-8 f_{3}^{L}(1)$. Thus (2.34) holds for all $m \in \mathbb{Z}$.

Case $3 \lambda=1$.

In this case (2.29) gives $(m-n)\left(f_{3}^{L}(m+n)-f_{3}^{L}(m)-f_{3}^{L}(n)\right)=0$. Using the similar discussions to the proof of (1), we have $f_{3}^{L}(m)=m f_{3}^{L}(1)$ for all $m \in \mathbb{Z}$.

Case $4 \lambda \notin\{-2, \pm 1,0\}$.

Letting $n=1$ in (2.29), we have

$$
(m-\lambda) f_{3}^{L}(m)-(1-\lambda m) f_{3}^{L}(1)=(m-1) f_{3}^{L}(m+1) .
$$

Replacing $m$ by $m+1, n$ by -1 in (2.29), we obtain

$$
(m+1+\lambda) f_{3}^{L}(m+1)+(1+\lambda(m+1)) f_{3}^{L}(-1)=(m+2) f_{3}^{L}(m) .
$$


Thus, using (2.36) and (2.37), one can deduce

$$
(1-\lambda)(2+\lambda) f_{3}^{L}(m)=(1-\lambda m)(m+1+\lambda) f_{3}^{L}(1)-(m-1)(\lambda(m+1)+1) f_{3}^{L}(-1) .
$$

Taking $m=-1$ in (2.38), we have $\lambda(1+\lambda)\left(f_{3}^{L}(1)+f_{3}^{L}(-1)\right)=0$, which forces $f_{3}^{L}(1)=$ $-f_{3}^{L}(-1)$. Then (2.38) gives $(1-\lambda)(\lambda+2)\left(f_{3}^{L}(m)-m f_{3}^{L}(1)\right)=0$. Thus $f_{3}^{L}(m)=m f_{3}^{L}(1)$ for all $m \in \mathbb{Z}$.

Hence denoted by $f_{1}^{L}(1)=a, f_{2}^{L}(1)=b, f_{2}^{L}(0)=e, f_{3}^{L}(0)=\bar{e}, f_{3}^{L}(2)=c$ and $f_{3}^{L}(1)=\bar{c}$, the lemma follows.

Lemma 2.3 Define $a, b$ and $e$ as those given in Lemma 2.2. For any $n \in \mathbb{Z}$ and some $\bar{a}, \hat{b}, \bar{b} \in \mathbb{C}$, we have

(1) $f_{1}^{Y}(n)=0$.

(2) $f_{2}^{Y}(n)=a n+\bar{a}$.

(3) If $\mu \notin \mathbb{Z}$, then $f_{3}^{Y}(n)=-\frac{e}{\mu} n$.

(4) If $\mu=0$, then

$$
f_{3}^{Y}(n)= \begin{cases}\hat{b} n & \text { if } \lambda=-1 \\ b n+\bar{b} & \text { if } \lambda=1 \\ \frac{2 b}{1+\lambda} n & \text { if } \lambda \neq \pm 1\end{cases}
$$

Proof (1) Applying $d$ to $\left[L_{n}, Y_{m}\right]=\left(m-\frac{1+\lambda}{2} n+\mu\right) Y_{m+n}$ and comparing the coefficients of $L_{m+n}$, we have

$$
2(m-n) f_{1}^{Y}(m)-(2 m-(1+\lambda) n+2 \mu) f_{1}^{Y}(n+m)=0 .
$$

Case $1 \mu \notin \mathbb{Z}$.

Setting $n=0$ in (2.39), we have $\mu f_{1}^{Y}(m)=0$, which gives $f_{1}^{Y}(m)=0$ for all $m \in \mathbb{Z}$.

Case $2 \mu=0$.

In this case (2.39) gives

$$
2(m-n) f_{1}^{Y}(m)-(2 m-(1+\lambda) n) f_{1}^{Y}(n+m)=0 .
$$

Setting $m=0$ in (2.40), we have

$$
(1+\lambda) n f_{1}^{Y}(n)=2 n f_{1}^{Y}(0)
$$

Subcase $1 \lambda \neq \pm 1$. 
By (2.41), we have

$$
n f_{1}^{Y}(n)=\frac{2}{1+\lambda} n f_{1}^{Y}(0), \quad \forall n \in \mathbb{Z} .
$$

Multiplying (2.40) by $m(n+m)$ and using (2.42), we deduce $(\lambda-1) m n(m+n) f_{1}^{Y}(0)=0$ for all $m, n \in \mathbb{Z}$, since $\lambda \neq 1$, which forces $f_{1}^{Y}(0)=0$. Using this in (2.42), we obtain $f_{1}^{Y}(n)=0$ for all $n \in \mathbb{Z}$.

Subcase $2 \lambda=-1$.

Setting $\lambda=-1$ in (2.40), we have

$$
(m-n) f_{1}^{Y}(m)-m f_{1}^{Y}(n+m)=0 .
$$

Replacing $n$ by $-m$ in (2.43), we have $f_{1}^{Y}(m)=\frac{1}{2} f_{1}^{Y}(0)$ for $m \neq 0$. Furthermore, letting $\lambda=-1$ in (2.41), we have $n f_{1}^{Y}(0)=0$ for all $n \in \mathbb{Z}$, which forces $f_{1}^{Y}(0)=0$. Thus $f_{1}^{Y}(m)=0$ for all $m \in \mathbb{Z}$.

Subcase $3 \lambda=1$.

Letting $\lambda=1$ in (2.40), we have

$$
(m-n)\left(f_{1}^{Y}(m)-f_{1}^{Y}(n+m)\right)=0 .
$$

Setting $m=0$ in (2.44), we obtain $n\left(f_{1}^{Y}(n)-f_{1}^{Y}(0)\right)=0$, which gives

$$
f_{1}^{Y}(n)=f_{1}^{Y}(0), \quad \forall n \in \mathbb{Z}
$$

Applying $d$ to $\left[Y_{2}, M_{3}\right]=0$ and comparing the coefficients of $M_{5}$, we have

$$
f_{2}^{M}(3)=-f_{1}^{Y}(2)=-f_{1}^{Y}(0)
$$

Furthermore, applying $d$ to $\left[Y_{1}, Y_{2}\right]=M_{3}$ and comparing the coefficients, we have $f_{2}^{M}(3)=$ $f_{1}^{Y}(1)+f_{1}^{Y}(2)$. This together with (2.45) and (2.46) gives $f_{1}^{Y}(0)=0$. Thus $f_{1}^{Y}(n)=0$ for all $n \in \mathbb{Z}$.

(2) From Lemma 2.2, we have $f_{1}^{L}(n)=$ an for some $a \in \mathbb{C}$. Thus applying $d$ to $\left[L_{n}, Y_{m}\right]=\left(m-\frac{1+\lambda}{2} n+\mu\right) Y_{m+n}$ and comparing the coefficient of $Y_{m+n}$, we have

$$
(2 m-(1+\lambda) n+2 \mu)\left(f_{2}^{Y}(m)-f_{2}^{Y}(n+m)+a n\right)=0 .
$$

Case $1 \lambda=-1$. 
In this case (2.47) gives

$$
(m+\mu)\left(f_{2}^{Y}(m)-f_{2}^{Y}(n+m)+a n\right)=0 .
$$

Recalling that $\mu$ satisfies (1.1), taking $m=1$ and replacing $n$ by $n-1$ in (2.48), we obtain

$$
f_{2}^{Y}(n)=a(n-1)+f_{2}^{Y}(1), \quad \forall n \in \mathbb{Z} .
$$

Taking $n=0$ in (2.49), we obtain $f_{2}^{Y}(1)=f_{2}^{Y}(0)+a$. Thus (2.49) gives $f_{2}^{Y}(n)=$ an $+f_{2}^{Y}(0)$ for all $n \in \mathbb{Z}$.

Case $2 \lambda \neq-1$.

If $\mu=0$, then setting $m=0$ in (2.47), we obtain $(1+\lambda) n\left(f_{2}^{Y}(0)-f_{2}^{Y}(n)+a n\right)=0$, which gives $f_{2}^{Y}(n)=a n+f_{2}^{Y}(0)$ for all $n \in \mathbb{Z}$.

Suppose $\mu \notin \mathbb{Z}$. Replacing $n$ by $-m$ in (2.47), we obtain

$$
((3+\lambda) m+2 \mu)\left(f_{2}^{Y}(m)-a m-f_{2}^{Y}(0)\right)=0 .
$$

If $(3+\lambda) m+2 \mu \neq 0$ for all $m \in \mathbb{Z}$, then (2.50) gives $f_{2}^{Y}(m)=a m+f_{2}^{Y}(0)$.

If $(3+\lambda) m^{\prime}+2 \mu=0$ for some $m^{\prime} \in \mathbb{Z}$, then replacing $m$ by $m^{\prime}+1$ in (2.50), we have

$$
(3+\lambda)\left(f_{2}^{Y}\left(m^{\prime}+1\right)-a\left(m^{\prime}+1\right)-f_{2}^{Y}(0)\right)=0 .
$$

It is obvious that $\lambda \neq-3$, otherwise $\mu=0$. Thus (2.51) gives

$$
f_{2}^{Y}\left(m^{\prime}+1\right)=a\left(m^{\prime}+1\right)+f_{2}^{Y}(0)
$$

Choosing $n=1$ and replacing $m$ by $m^{\prime}$ in (2.47), we obtain

$$
\left(2 \mu-\lambda+2 m^{\prime}-1\right)\left(a+f_{2}^{Y}\left(m^{\prime}\right)-f_{2}^{Y}\left(m^{\prime}+1\right)\right)=0 .
$$

Combining (2.52) and (2.53), one can deduce

$$
\left(2 \mu-\lambda+2 m^{\prime}-1\right)\left(f_{2}^{Y}\left(m^{\prime}\right)-a m^{\prime}-f_{2}^{Y}(0)\right)=0 .
$$

Furthermore, using $2 \mu=-(3+\lambda) m^{\prime}$ in (2.54), we have

$$
\left(m^{\prime}+1\right)(1+\lambda)\left(f_{2}^{Y}\left(m^{\prime}\right)-a m^{\prime}-f_{2}^{Y}(0)\right)=0 .
$$

In this case $\lambda \neq-1$, we obtain

$$
\left(m^{\prime}+1\right)\left(f_{2}^{Y}\left(m^{\prime}\right)-a m^{\prime}-f_{2}^{Y}(0)\right)=0
$$


If $m^{\prime} \neq-1$, then (2.56) gives $f_{2}^{Y}\left(m^{\prime}\right)=a m^{\prime}+f_{2}^{Y}(0)$.

Suppose that $m^{\prime}=-1$. Then $2 \mu=3+\lambda$. Setting $m=0, n=-1$ in (2.47), we obtain

$$
(\lambda+2)\left(f_{2}^{Y}(0)-f_{2}^{Y}(-1)-a\right)=0 .
$$

If $\lambda \neq-2$, then (2.57) forces $f_{2}^{Y}(-1)=-a+f_{2}^{Y}(0)$. If $\lambda=-2$, then setting $\lambda=-2$, $m=-1$ and $n=1$ in (2.47), we obtain

$$
(2 \mu-1)\left(a+f_{2}^{Y}(-1)-f_{2}^{Y}(0)\right)=0,
$$

since $\mu$ satisfies (1.1), which forces $f_{2}^{Y}(-1)=-a+f_{2}^{Y}(0)$. Hence $f_{2}^{Y}(m)=a m+f_{2}^{Y}(0)$ for all $m \in \mathbb{Z}$. By now we have completed the proof of (2).

Next we begin the proof of (3) and (4) of this lemma. Applying $d$ to $\left[L_{n}, Y_{m}\right]=(m-$ $\left.\frac{1+\lambda}{2} n+\mu\right) Y_{m+n}$ and comparing the coefficients of $M_{m+n}$, we have

$$
2(m-n) f_{2}^{L}(n)+2(m-\lambda n+2 \mu) f_{3}^{Y}(m)=(2 m-(1+\lambda) n+2 \mu) f_{3}^{Y}(n+m) .
$$

(3) If $\mu \notin \mathbb{Z}$, then taking $n=0$ in (2.59), we obtain $f_{3}^{Y}(m)=-\frac{e}{\mu} m$ for $e=f_{2}^{L}(0)$.

(4) Taking $\mu=0$ in (2.59), we obtain

$$
2(m-n) f_{2}^{L}(n)+2(m-\lambda n) f_{3}^{Y}(m)=(2 m-(1+\lambda) n) f_{3}^{Y}(n+m) .
$$

By Lemma $2.2(2)$, one has $f_{2}^{L}(n)=b n\left(1-\delta_{\lambda,-1}\right)$ for some $b \in \mathbb{C}$.

Case $1 \lambda=-1$.

In this case (2.60) gives

$$
(m+n) f_{3}^{Y}(m)=m f_{3}^{Y}(m+n) .
$$

Taking $m=1$ and replacing $n$ by $n-1$ in (2.61), we have $f_{3}^{Y}(n)=n f_{3}^{Y}(1)$ for all $n \in \mathbb{Z}$.

Case $2 \lambda=1$.

Letting $\lambda=1$ in (2.60), we obtain

$$
(m-n)\left(f_{3}^{Y}(n+m)-f_{3}^{Y}(m)-b n\right)=0 .
$$

Thus (2.62) gives $f_{3}^{Y}(n)=b n+f_{3}^{Y}(0)$ for all $n \in \mathbb{Z}$.

Case $3 \lambda \neq \pm 1$.

Setting $m=0$ in (2.60), we have

$$
f_{3}^{Y}(n)=\frac{2}{1+\lambda}\left(b n+\lambda f_{3}^{Y}(0)\right) \text { for } n \neq 0 .
$$


Furthermore, taking $n= \pm 1$ in (2.63) respectively, we have

$$
f_{3}^{Y}(1)=\frac{2}{1+\lambda}\left(\lambda f_{3}^{Y}(0)+b\right), \quad f_{3}^{Y}(-1)=\frac{2}{1+\lambda}\left(\lambda f_{3}^{Y}(0)-b\right) .
$$

Taking $n=1, m=-1$ in (2.60) and using (2.64), one can deduce $(\lambda-1) f_{3}^{Y}(0)=0$, which forces $f_{3}^{Y}(0)=0$ since $\lambda \neq 1$. Then (2.63) implies $f_{3}^{Y}(n)=\frac{2 b}{1+\lambda} n$ for all $n \in \mathbb{Z}$.

Hence, denoted by $f_{2}^{Y}(0)=\bar{a}, f_{3}^{Y}(1)=\hat{b}$ and $f_{3}^{Y}(0)=\bar{b}$, the lemma follows.

Lemma 2.4 Let $a$ and $\bar{a}$ be as those given in Lemmas 2.2 and 2.3 respectively. For any $n \in \mathbb{Z}$, we have

$$
f_{1}^{M}(n)=f_{2}^{M}(n)=0, \quad f_{3}^{M}(n)=a n+2 \bar{a} .
$$

Proof It follows from Lemmas 2.2 and 2.3 that there exists some $a$ and $\bar{a}$ such that

$$
f_{1}^{Y}(n)=0, \quad f_{2}^{Y}(n)=a n+\bar{a}, \quad \forall n \in \mathbb{Z} .
$$

Applying $d$ to $\left[Y_{n}, Y_{m}\right]=(m-n) M_{m+n}$ and comparing the coefficients of $L_{m+n}, Y_{m+n}$ and $M_{m+n}$ respectively, we have

$$
\begin{aligned}
& (m-n) f_{1}^{M}(m+n)=(m-n) f_{2}^{M}(m+n)=0, \\
& (m-n)\left(f_{3}^{M}(m+n)-a(n+m)-2 \bar{a}\right)=0 .
\end{aligned}
$$

Setting $m=0$ and replacing $m$ by $-n$ in (2.65), we obtain

$$
n f_{1}^{M}(n)=n f_{2}^{M}(n)=0, \quad n f_{1}^{M}(0)=n f_{2}^{M}(0)=0, \quad \forall n \in \mathbb{Z} .
$$

This gives $f_{1}^{M}(n)=f_{2}^{M}(n)=0$ for all $n \in \mathbb{Z}$.

Taking $m=0$ in (2.66), one can deduce $f_{3}^{M}(n)=a n+2 \bar{a}$ for $n \neq 0$. Taking $m=1$ and $n=-1$ in (2.66), one can deduce $f_{3}^{M}(0)=2 \bar{a}$. Thus $f_{3}^{M}(n)=a n+2 \bar{a}$ for all $n \in \mathbb{Z}$.

We construct some possible outer derivations of $\mathscr{L}$. Under the condition $\mu=0$, for each $\lambda \in\{-2, \pm 1\}$, the following maps $D_{\lambda}$ or $\bar{D}_{\lambda}$ defined by

$$
D_{-2}\left(L_{n}\right)=n^{3} M_{n}, D_{-1}\left(L_{n}\right)=n^{2} M_{n}, \bar{D}_{-1}\left(Y_{n}\right)=n M_{n}, D_{1}\left(Y_{n}\right)=M_{n},
$$

are outer derivations of $\mathscr{L}$, where all other terms are vanishing. Besides, we can define another outer derivation $D$ of $\mathscr{L}$, which does not depend on $\lambda$ and $\mu$ :

$$
D: L_{n} \mapsto 0, Y_{n} \mapsto Y_{n}, M_{n} \mapsto 2 M_{n}
$$

It is easy to verity that for each $\lambda \in\{-2, \pm 1\}, D, D_{\lambda}$ and $\bar{D}_{\lambda}$ are linearly independent. 
Theorem 2.5 (1) If $\mu \notin \frac{1}{2} \mathbb{Z}$, or $\mu=0$ but $\lambda \notin\{-2,0, \pm 1\}$, then Der $\mathscr{L}=$ ad $\mathscr{L} \oplus \mathbb{C} D$.

(2) If $\mu=0$, then

$$
\operatorname{Der} \mathscr{L}= \begin{cases}a d \mathscr{L} \oplus \mathbb{C} D \oplus \mathbb{C} D_{-2}, & \lambda=-2, \\ \operatorname{ad} \mathscr{L} \oplus \mathbb{C} D \oplus \mathbb{C} D_{-1} \oplus \mathbb{C} \bar{D}_{-1}, & \lambda=-1, \\ \operatorname{ad} \mathscr{L} \oplus \mathbb{C} D \oplus \mathbb{C} D_{1}, & \lambda=1 .\end{cases}
$$

Proof Take $d_{0} \in(\operatorname{Der} \mathscr{L})_{0}$.

(1) Suppose $\mu \notin \frac{1}{2} \mathbb{Z}$. It follows from Lemmas 2.22 .4 that there exist $a, \bar{a}, e$ and $\bar{e} \in \mathbb{C}$ such that

$$
\begin{aligned}
& d_{0}\left(L_{n}\right)=a n L_{n}+\frac{e}{2 \mu}(2 \mu-(\lambda+1) n) Y_{n}+\frac{\bar{e}}{2 \mu}(2 \mu-\lambda n) M_{n} \\
& d_{0}\left(Y_{n}\right)=(a n+\bar{a}) Y_{n}-\frac{e}{\mu} n M_{n}, \quad d_{0}\left(M_{n}\right)=(a n+2 \bar{a}) M_{n} .
\end{aligned}
$$

Set $\alpha=a L_{0}-e \mu^{-1} Y_{0}-\bar{e}(2 \mu)^{-1} M_{0}$, then $d_{0}=a d_{\alpha}+(\bar{a}-a \mu) D$.

If $\mu=0$ and $\lambda \notin\{-2,0, \pm 1\}$, by Lemmas 2.2 2.4, there exist $a^{\prime}, \bar{a}^{\prime}, b^{\prime}$ and $e^{\prime} \in \mathbb{C}$ such that

$$
\begin{aligned}
& d_{0}\left(L_{n}\right)=a^{\prime} n L_{n}+b^{\prime} n Y_{n}+e^{\prime} n M_{n}, \\
& d_{0}\left(Y_{n}\right)=\left(a^{\prime} n+\bar{a}^{\prime}\right) Y_{n}+\frac{2 b^{\prime}}{1+\lambda} n M_{n}, \quad d_{0}\left(M_{n}\right)=\left(a^{\prime} n+2 \bar{a}^{\prime}\right) M_{n} .
\end{aligned}
$$

Set $\beta=a^{\prime} L_{0}+2 b^{\prime}(1+\lambda)^{-1} Y_{0}+e^{\prime} \lambda^{-1} M_{0}$. Then $d_{0}=a d_{\beta}+\bar{a}^{\prime} D$.

(2) We shall divide the proof of (2.67) into the following four cases.

Case $1 \lambda=-2$.

By Lemmas 2.2 2.4, there exist $a_{1}, \bar{a}_{1}, b_{1}, c_{1}$ and $\bar{c}_{1} \in \mathbb{C}$ such that

$$
\begin{aligned}
& d_{0}\left(L_{n}\right)=a_{1} n L_{n}+b_{1} n Y_{n}+\left(\frac{c_{1}}{6}\left(n^{3}-n\right)-\frac{\bar{c}_{1}}{3}\left(n^{3}-4 n\right)\right) M_{n}, \\
& d_{0}\left(Y_{n}\right)=\left(a_{1} n+\bar{a}_{1}\right) Y_{n}-2 b_{1} n M_{n}, \quad d_{0}\left(M_{n}\right)=\left(a_{1} n+2 \bar{a}_{1}\right) M_{n} .
\end{aligned}
$$

Set $\alpha_{1}=a_{1} L_{0}-2 b_{1} Y_{0}+\frac{1}{12}\left(c_{1}-8 \bar{c}_{1}\right) M_{0}$. Then we obtain $d_{0}=a d_{\alpha_{1}}+\bar{a}_{1} D+\frac{1}{6}\left(c_{1}-2 \bar{c}_{1}\right) D_{-2}$.

Case $2 \lambda=-1$.

By Lemmas 2.2 2.4, there exist $a_{2}, \bar{a}_{2}, b_{2}, c_{2}$ and $\bar{c}_{2} \in \mathbb{C}$ such that

$$
\begin{aligned}
& d_{0}\left(L_{n}\right)=a_{2} n L_{n}+\left(\frac{c_{2}}{2}\left(n^{2}-n\right)-\bar{c}_{2}\left(n^{2}-2 n\right)\right) M_{n}, \\
& d_{0}\left(Y_{n}\right)=\left(a_{2} n+\bar{a}_{2}\right) Y_{n}+b_{2} n M_{n}, \quad d_{0}\left(M_{n}\right)=\left(a_{2} n+2 \bar{a}_{2}\right) M_{n} .
\end{aligned}
$$

Set $\alpha_{2}=a_{2} L_{0}+\frac{1}{2}\left(c_{2}-4 \bar{c}_{2}\right) M_{0}$. Then $d_{0}=a d_{\alpha_{2}}+\bar{a}_{2} D+\frac{1}{2}\left(c_{2}-2 \bar{c}_{2}\right) D_{-1}+b_{2} \bar{D}_{-1}$. 
Case $3 \lambda=1$.

By Lemmas 2.2 2.4, there exist $a_{4}, \bar{a}_{4}, b_{4}, \bar{b}$ and $c_{4} \in \mathbb{C}$ such that

$$
\begin{aligned}
& d_{0}\left(L_{n}\right)=a_{4} n L_{n}+b_{4} n Y_{n}+c_{4} n M_{n}, \\
& d_{0}\left(Y_{n}\right)=\left(a_{4} n+\bar{a}_{4}\right) Y_{n}+\left(b_{4} n+\bar{b}\right) M_{n}, \quad d_{0}\left(M_{n}\right)=\left(a_{4} n+2 \bar{a}_{4}\right) M_{n} .
\end{aligned}
$$

Set $\alpha_{4}=a_{4} L_{0}+b_{4} Y_{0}+c_{4} M_{0}$. Then $d_{0}=a d_{\alpha_{4}}+\bar{a}_{4} D+\bar{b} D_{1}$.

Hence, the theorem follows from Lemma 2.1.

\section{Automorphism group of $\mathscr{L}$}

In this section we denote by $\mathbb{C}^{*}$ the set of all nonzero complex numbers and Aut $\mathscr{L}$ and $\operatorname{Inn} \mathscr{L}$ the sets of automorphisms and inner automorphisms of $\mathscr{L}$. Firstly we need to introduce some technical lemmas.

Lemma 3.1 Let $\sigma \in$ Aut $\mathscr{L}, \epsilon \in\{ \pm 1\}$ with $\epsilon=1$ for $\mu \notin \frac{1}{2} \mathbb{Z}$. Then there exist some $\alpha$, $\beta \in \mathbb{C}^{*}, y_{n} \in \mathcal{Y}$ and $m_{n}, m_{n}^{\prime} \in \mathcal{M}$ such that

(1) $\sigma\left(L_{n}\right)=\epsilon \alpha^{n} L_{\epsilon n}+y_{n}+m_{n}$

(2) $\sigma\left(Y_{n}\right)=\alpha^{n} \beta Y_{\epsilon n}+m_{n}^{\prime}$,

(3) $\sigma\left(M_{n}\right)=\epsilon \alpha^{n} \beta^{2} M_{\epsilon n}$.

Proof (1) Note that $\left.\sigma\right|_{\mathcal{L}}$ is an automorphism of the Witt algebra, so there exist some $\epsilon \in\{ \pm 1\}$ and $\alpha \in \mathbb{C}^{*}$ such that

$$
\sigma\left(L_{n}\right)=\epsilon \alpha^{n} L_{\epsilon n}+y_{n}+m_{n} \quad \text { for some } y_{n} \in \mathcal{Y}, m_{n} \in \mathcal{M}
$$

(2) Since $\mathcal{I}=\mathcal{Y} \oplus \mathcal{M}$ is the unique maximal idea of $\mathscr{L}$, one can write

$$
\sigma\left(Y_{n}\right)=\sum_{i \in S} b_{n_{i}} Y_{i}+m_{n}^{\prime}
$$

for some $b_{n_{i}} \in \mathbb{C}^{*}, m_{n}^{\prime} \in \mathcal{M}$ and $S \subseteq \mathbb{Z}$. By (3.1), there exist some $y_{0} \in \mathcal{Y}, m_{0} \in \mathcal{M}$ such that $\sigma\left(L_{0}\right)=\epsilon L_{0}+y_{0}+m_{0}$. Then applying $\sigma$ to $(n+\mu) Y_{n}=\left[L_{0}, Y_{n}\right]$, we have

$$
(n+\mu)\left(\sum_{i \in S} b_{n_{i}} Y_{i}+m_{n}^{\prime}\right)=\left[\epsilon L_{0}+y_{0}+m_{0}, \sum_{i \in S} b_{n_{i}} Y_{i}+m_{n}^{\prime}\right]=\sum_{i \in S} \epsilon b_{n_{i}}(i+\mu) Y_{i}+m_{n}^{\prime \prime},
$$

for some $m_{n}^{\prime \prime} \in \mathcal{M}$. Comparing the coefficients of $Y_{i}$, we obtain

$$
b_{n_{i}}(i-\epsilon n-(\epsilon-1) \mu)=0, \forall i \in S
$$

If $\mu=0$, then (3.3) gives $i=\epsilon n$ for all $i \in S$. If $\mu \notin \frac{1}{2} \mathbb{Z}$, then (3.3) forces $\epsilon=1$, in which case (3.3) gives $i=n$ for all $i \in S$. Hence, by (3.2), we can write

$$
\sigma\left(Y_{n}\right)=b_{n} Y_{\epsilon n}+m_{n}^{\prime}
$$


for some $b_{n} \in \mathbb{C}^{*}$ with $\epsilon=1$ for $\mu \notin \frac{1}{2} \mathbb{Z}$.

Applying $\sigma$ to $\left(m-\frac{\lambda+1}{2} n+\mu\right) Y_{m+n}=\left[L_{n}, Y_{m}\right]$ and using (3.1) and (3.4), we obtain

$$
\begin{aligned}
& \left(m-\frac{\lambda+1}{2} n+\mu\right)\left(b_{m+n} Y_{\epsilon(m+n)}+m_{m+n}^{\prime}\right) \\
= & {\left[\epsilon \alpha^{n} L_{\epsilon n}+y_{n}+m_{n}, b_{m} Y_{\epsilon m}+m_{m}^{\prime}\right]=\alpha^{n} b_{m}\left(m-\frac{\lambda+1}{2} n+\mu\right) Y_{\epsilon(m+n)}+\bar{m}_{n}, }
\end{aligned}
$$

for some $\bar{m}_{n} \in \mathcal{M}$. Thus we have

$$
\left(m-\frac{\lambda+1}{2} n+\mu\right)\left(\left(b_{m+n}-\alpha^{n} b_{m}\right) Y_{\epsilon(m+n)}+m_{m+n}^{\prime}\right)-\bar{m}_{n}=0,
$$

which together with the fact $m_{m+n}^{\prime}, \bar{m}_{n} \in \mathcal{M}$, gives

$$
(2 m-(\lambda+1) n+2 \mu)\left(b_{m+n}-\alpha^{n} b_{m}\right)=0 .
$$

Taking $m=0$ in (3.5), one has

$$
(2 \mu-(\lambda+1) n)\left(b_{n}-\alpha^{n} b_{0}\right)=0 .
$$

If $2 \mu-(\lambda+1) n^{\prime}=0$ for some $n^{\prime} \in \mathbb{Z}$, then replacing $n$ by $-n^{\prime}, m$ by $n^{\prime}$ in (3.5), we have

$$
(\lambda+2) n^{\prime}\left(b_{n^{\prime}}-\alpha^{n^{\prime}} b_{0}\right)=0 .
$$

If $\lambda \neq-2$, then (3.7) gives $b_{n^{\prime}}=\alpha^{n^{\prime}} b_{0}$.

If $\lambda=-2$, then $2 \mu+n^{\prime}=0$, since $\mu$ satisfies (1.1), which forces $n^{\prime}=0$. Thus

$$
b_{n^{\prime}}=\alpha^{n^{\prime}} b_{0} \quad \text { if } 2 \mu-(\lambda+1) n^{\prime}=0 .
$$

Hence, (3.6) together with (3.8) gives $b_{n}=\alpha^{n} b_{0}$ for all $n \in \mathbb{Z}$. Using this in (3.4), one can write

$$
\sigma\left(Y_{n}\right)=\alpha^{n} b_{0} Y_{\epsilon n}+m_{n}^{\prime}
$$

for some $m_{n}^{\prime} \in \mathcal{M}$.

(3) Applying $\sigma$ to $(n-2 m) M_{n}=\left[Y_{m}, Y_{n-m}\right]$ and using (3.9), we have $(n-2 m) \sigma\left(M_{n}\right)=$ $\left[\alpha^{m} b_{0} Y_{\epsilon m}+m_{m}^{\prime}, \alpha^{n-m} b_{0} Y_{\epsilon(n-m)}+m_{n-m}^{\prime}\right]$ for some $m_{m}^{\prime}, m_{n-m}^{\prime} \in \mathcal{M}$, which gives

$$
(n-2 m)\left(\sigma\left(M_{n}\right)-\epsilon \alpha^{n} b_{0}^{2} M_{\epsilon n}\right)=0 .
$$

Letting $m=0$ in (3.10), we have $\sigma\left(M_{n}\right)=\epsilon \alpha^{n} b_{0}^{2} M_{\epsilon n}$ for $n \neq 0$. Furthermore, letting $n=0$ in (3.10), one has $\sigma\left(M_{0}\right)=\epsilon b_{0}^{2} M_{0}$. Thus we obtain $\sigma\left(M_{n}\right)=\epsilon \alpha^{n} b_{0}^{2} M_{\epsilon n}$.

Hence, denoted by $b_{0}=\beta$, the lemma follows. 
Lemma 3.2 Let $\bar{f}, f$ and $g$ be $\mathbb{C}$-linear maps from $\mathbb{Z}$ to $\mathbb{C}$. Define the $\mathbb{C}$-linear map $\phi$ : $\mathscr{L} \rightarrow \mathscr{L}$ by

$$
\phi\left(L_{n}\right)=L_{n}+\bar{f}(n) Y_{n}+f(n) M_{n}, \phi\left(Y_{n}\right)=Y_{n}+g(n) M_{n}, \phi\left(M_{n}\right)=M_{n} .
$$

If $\mu=0$ and $\phi \in A u t \mathscr{L}$, then there exist some $a, \bar{a}, b, c, \bar{c} \in \mathbb{C}$ such that

(1)

$$
\bar{f}(n)=b n\left(1-\delta_{\lambda,-1}\right), \quad g(n)= \begin{cases}a n & \text { if } \lambda=-1, \\ b n+\bar{a} & \text { if } \lambda=1 \\ \frac{2 b}{1+\lambda} n & \text { if } \lambda \neq \pm 1\end{cases}
$$

$$
f(n)= \begin{cases}\frac{1}{6} c\left(n^{3}-n\right)-\frac{1}{3} \bar{c}\left(n^{3}-4 n\right)+\frac{1}{3} b^{2} n(n-2)(n-1) & \text { if } \lambda=-2, \\ \frac{1}{2} c\left(n^{2}-n\right)-\bar{c}\left(n^{2}-2 n\right) & \text { if } \lambda=-1, \\ \bar{c} n+\frac{b^{2}}{1+\lambda} n(n-1) & \text { if } \lambda \notin\{-2,0,-1\} .\end{cases}
$$

Proof Applying $\phi$ to $\left[L_{n}, L_{m}\right]=(m-n) L_{n+m}$ and comparing the coefficients of $Y_{n+m}$, $M_{n+m}$, we obtain

$$
\begin{aligned}
& (2 m-(1+\lambda) n) \bar{f}(m)-(2 n-(1+\lambda) m) \bar{f}(n)=2(m-n) \bar{f}(m+n), \\
& (m-\lambda n) f(m)-(n-\lambda m) f(n)=(m-n)(f(m+n)-\bar{f}(n) \bar{f}(m)) .
\end{aligned}
$$

Applying $\phi$ to $\left[L_{n}, Y_{m}\right]=\left(m-\frac{\lambda+1}{2} n\right) Y_{n+m}$ and comparing the coefficients of $M_{n+m}$, we obtain

$$
2(m-n) \bar{f}(n)+2(m-\lambda n) g(m)=(2 m-(\lambda+1) n) g(n+m) .
$$

If we replace $\bar{f}$ by $f_{2}^{L}, g$ by $f_{3}^{Y}$ in (3.11) and (3.13), then (3.11) and (3.13) become (2.3) and (2.60), respectively. Thus if we take $\bar{f}(1)=b, g(1)=a$ and $g(0)=\bar{a}$, then by Lemmas 2.2 and 2.3. (1) follows.

(2) If $\lambda=-1$, then noticing $\bar{f}(n)=0$ in (3.12), we obtain

$$
(m+n) f(m)-(n+m) f(n)=(m-n) f(m+n) .
$$

If we replace $f_{3}^{L}$ by $f, \lambda$ by -1 in (2.29), then (2.29) becomes (3.14). Thus by Lemma 2.2 (3), we have $f(n)=\frac{1}{2}\left(n^{2}-n\right) f(2)-\left(n^{2}-2 n\right) f(1)$.

If $\lambda \neq-1$, noticing $\bar{f}(n)=b n$ in (3.12), we have

$$
(m-\lambda n) f(m)-(n-\lambda m) f(n)=(m-n)\left(f(m+n)-b^{2} n m\right) .
$$


Taking $n=1,2$ in (3.15) respectively, we have

$$
\begin{aligned}
& (m-\lambda) f(m)-(1-\lambda m) f(1)=(m-1)\left(f(m+1)-b^{2} m\right), \\
& (m-2 \lambda) f(m)-(2-\lambda m) f(2)=(m-2)\left(f(m+2)-2 b^{2} m\right) .
\end{aligned}
$$

Setting $n=1$ and replacing $m$ by $m+1$ in (3.15), one has

$$
(m+1-\lambda) f(m+1)-(1-\lambda(m+1)) f(1)=m\left(f(m+2)-b^{2}(m+1)\right) .
$$

Thus using (3.16)-(3.18), one can deduce

$$
\begin{aligned}
& (\lambda-1)((\lambda+2) m-2 \lambda) f(m)-b^{2}(\lambda-2) m(m-2)(m-1) \\
& =m(m-1)(\lambda m-2) f(2)+m(m-2)\left(2-\lambda+\lambda^{2}-2 \lambda m\right) f(1) .
\end{aligned}
$$

Case $1 \lambda=-2$.

In this case (3.19) gives

$$
f(m)=\frac{1}{6}\left(m^{3}-m\right) f(2)-\frac{1}{3}\left(m^{3}-4 m\right) f(1)+\frac{b^{2}}{3} m(m-2)(m-1), \quad \forall m \in \mathbb{Z} .
$$

Case $2 \lambda=1$.

In this case (3.15) gives

$$
(m-n)\left(f(m)+f(n)-f(m+n)+b^{2} n m\right)=0 .
$$

Obviously, $f(0)=0$. Replacing $m$ by $-n$ in (3.20), one can deduce

$$
f(-n)+f(n)=b^{2} n^{2}, \forall n \in \mathbb{Z}
$$

By (3.20), we have

$$
f(m+n)=f(m)+f(n)+b^{2} n m \text { for } m \neq n .
$$

Combining (3.21), (3.22), we obtain

$$
\begin{aligned}
f(2 m) & =f(3 m)+f(-m)-3 b^{2} m^{2} \\
& =f(2 m)+f(m)+2 b^{2} m^{2}+f(-2 m)+f(m)-2 b^{2} m^{2}-3 b^{2} m^{2} \\
& =2 f(m)+b^{2} m^{2} .
\end{aligned}
$$

Thus (3.22) holds for all $m, n \in \mathbb{Z}$. Taking $m=1$ and replacing $n$ by $n-1$ in (3.22), we obtain $f(n)=f(n-1)+f(1)+b^{2}(n-1)$. Hence, using induction on $n$, one can deduce $f(n)=n f(1)+\frac{1}{2} b^{2} n(n-1)$. 
Case $3 \lambda \notin\{-2,0,1\}$.

Taking $m=0$ in (3.15), one has $\lambda n f(0)=0$ for all $n \in \mathbb{Z}$. Thus $f(0)=0$. Using this, then setting $m=-1$ and $n=1$ in (3.15), one can deduce

$$
(1+\lambda) f(-1)=2 b^{2}-(1+\lambda) f(1)
$$

Then taking $n=1$ in (3.15), we have

$$
(m-1) f(m+1)=(m-\lambda) f(m)-(1-\lambda m) f(1)+(m-1) b^{2} m .
$$

Setting $n=-1$ and replacing $m$ by $m+1$ in (3.15), we obtain

$$
(m+1+\lambda) f(m+1)+(1+\lambda(m+1)) f(-1)=(m+2)\left(f(m)+b^{2}(m+1)\right) .
$$

Multiplying (3.25) by $(1+\lambda)(m-1)$, then using (3.23) and (3.24), one can deduce

$$
\begin{aligned}
& (1-\lambda)(1+\lambda)(2+\lambda) f(m) \\
= & (1-\lambda)(1+\lambda)(2+\lambda) m f(1)+b^{2}(1-\lambda)(2+\lambda) m(m-1),
\end{aligned}
$$

since $\lambda \notin\{-2, \pm 1\}$, which gives $f(m)=m f(1)+\frac{b^{2}}{\lambda+1} m(m-1)$ for all $m \in \mathbb{Z}$.

Thus denoted by $f(2)=c$ and $f(1)=\bar{c}$, the lemma follows.

Lemma 3.3 (i) Let $\epsilon \in\{ \pm 1\}$. If $\mu=0$, then the map

$$
\varphi_{\epsilon}: L_{n} \mapsto \epsilon L_{\epsilon n}, Y_{n} \mapsto Y_{\epsilon n}, M_{n} \mapsto \epsilon M_{\epsilon n}
$$

is an automorphism of $\mathscr{L}$. The set $\left\{\varphi_{\epsilon} \mid \epsilon \in\{ \pm 1\}\right\} \cong \mathbb{Z}_{2}$ forms a subgroup of Aut $\mathscr{L}$, where $\varphi_{\epsilon} \varphi_{\epsilon^{\prime}}=\varphi_{\epsilon \epsilon^{\prime}}$ for $\epsilon, \epsilon^{\prime} \in\{ \pm 1\}$.

(ii) For any $\alpha, \beta \in \mathbb{C}^{*}$, the map

$$
\varphi_{\alpha, \beta}: L_{n} \mapsto \alpha^{n} L_{n}, Y_{n} \mapsto \alpha^{n} \beta Y_{n}, M_{n} \mapsto \alpha^{n} \beta^{2} M_{n}
$$

is an automorphism of $\mathscr{L}$. The set $\left\{\varphi_{\alpha, \beta} \mid \alpha, \beta \in \mathbb{C}^{*}\right\} \cong \mathbb{C}^{*} \times \mathbb{C}^{*}$ forms a subgroup of Aut $\mathscr{L}$, where $\varphi_{\alpha, \beta} \varphi_{\alpha^{\prime}, \beta^{\prime}}=\varphi_{\alpha \alpha^{\prime}, \beta \beta^{\prime}}$ for $\alpha, \alpha^{\prime}, \beta, \beta^{\prime} \in \mathbb{C}^{*}$.

(iii) For any $b \in \mathbb{C}$, if $\mu=0$, then the map

$$
\phi_{b}\left(L_{n}\right)=\left\{\begin{array}{ll}
L_{n}+b n^{3} M_{n}, & \lambda=-2, \\
L_{n}+b n^{2} M_{n}, & \lambda=-1,
\end{array} \phi_{b}\left(X_{n}\right)=X_{n} \quad \text { for } X \in\{Y, M\},\right.
$$

is an automorphism of $\mathscr{L}$. The set $\left\{\phi_{b} \mid b \in \mathbb{C}\right\} \cong \mathbb{C}$ forms a subgroup of Aut $\mathscr{L}$, where $\phi_{b} \phi_{b^{\prime}}=\phi_{b+b^{\prime}}$ for $b, b^{\prime} \in \mathbb{C}$. 
(iv) For any $e \in \mathbb{C}$, if $\mu=0$, then the map $\psi_{e}$ defined by

$$
\begin{aligned}
& L_{n} \mapsto L_{n}, \quad Y_{n} \mapsto Y_{n}+e n M_{n}, \quad M_{n} \mapsto M_{n}, \quad \lambda=-1, \\
& L_{n} \mapsto L_{n}, \quad Y_{n} \mapsto Y_{n}+e M_{n}, \quad M_{n} \mapsto M_{n}, \quad \lambda=1,
\end{aligned}
$$

is an automorphism of $\mathscr{L}$. The set $\left\{\psi_{e} \mid e \in \mathbb{C}\right\} \cong \mathbb{C}$ forms a subgroup of Aut $\mathscr{L}$, where $\psi_{e} \psi_{e^{\prime}}=\psi_{e+e^{\prime}}$ for $e, e^{\prime} \in \mathbb{C}$.

Proof This follows from straightforward verifications, we omit the details here.

Introduce the following notation

$$
\operatorname{Inn} \mathscr{L}=\operatorname{Span}\left\{\exp \left(a \operatorname{ad} L_{0}+\sum b_{i} \operatorname{ad} Y_{i}+\sum c_{j} \operatorname{ad} M_{j}\right) \mid a, b_{i}, c_{j} \in \mathbb{C}, i, j \in \mathbb{Z}\right\}
$$

with $a=0$ if $\mu \notin \mathbb{Z}$.

Theorem 3.4 (1) If $\mu \notin \frac{1}{2} \mathbb{Z}$, then Aut $\mathscr{L} \cong \operatorname{Inn} \mathscr{L} \times \mathbb{C}^{*} \times \mathbb{C}^{*}$.

(2) If $\mu=0$, then

$$
\text { Aut } \mathscr{L} \cong \begin{cases}\operatorname{Inn} \mathscr{L} \times \mathbb{C}^{*} \times \mathbb{Z}_{2} \times \mathbb{C} & \text { if } \lambda=-2 \text { or } 1, \\ \operatorname{Inn} \mathscr{L} \times \mathbb{C}^{*} \times \mathbb{Z}_{2} \times \mathbb{C} \times \mathbb{C} & \text { if } \lambda=-1 \\ \operatorname{Inn} \mathscr{L} \times \mathbb{C}^{*} \times \mathbb{Z}_{2} & \text { if } \lambda \notin\{-2,0, \pm 1\}\end{cases}
$$

Proof Let $\sigma$ be an automorphism of $\mathscr{L}$. By Lemma 3.1, one can write

$$
\sigma\left(L_{0}\right)=\epsilon L_{0}+b_{0} Y_{0}+c_{0} M_{0}+\sum_{0 \neq i \in S} b_{i} Y_{i}+\sum_{0 \neq j \in S^{\prime}} c_{j} M_{j}
$$

for some $S, S^{\prime} \subset \mathbb{Z}$. Construct an inner automorphism $\theta$ of $\mathscr{L}$

$$
\theta=\exp \left(\sum_{0 \neq i \in S} \frac{i b_{0} b_{i}}{(i+\mu)(i+2 \mu)} \operatorname{ad} M_{i}-\frac{b_{i}}{\epsilon(i+\mu)} \operatorname{ad} Y_{i}\right) \exp \left(\sum_{0 \neq j \in S^{\prime}} \frac{-c_{j}}{\epsilon(j+2 \mu)} \operatorname{ad} M_{j}\right)
$$

One can check that $\theta^{-1} \sigma\left(L_{0}\right)=\epsilon L_{0}+b_{0} Y_{0}+c_{0} M_{0}$. Furthermore, since $L_{0}$ is a semisimple element of $\mathscr{L}$, then $\theta^{-1} \sigma\left(L_{0}\right)$ is also semisimple. If we denote $A$ the matrix of $\operatorname{ad}\left(\theta^{-1} \sigma\left(L_{0}\right)\right)$ with respect to the basis $\left\{L_{n}, Y_{n}, M_{n}\right\}$, then

$$
A=\left(\begin{array}{ccc}
\epsilon n & b_{0}\left(\frac{\lambda+1}{2} n-\mu\right) & c_{0}(\lambda n-2 \mu) \\
0 & \epsilon(n+\mu) & b_{0} n \\
0 & 0 & \epsilon(n+2 \mu)
\end{array}\right)
$$

On the other hand, it follows from the definition of semisimple that $A$ can be diagonalized. Thus it is necessary that $b_{0}$ and $c_{0}(\lambda n-2 \mu)$ are equal to 0 . 
By Lemma 3.1, one can write

$$
\left\{\begin{array}{l}
\theta^{-1} \sigma\left(L_{n}\right)=\epsilon \bar{\alpha}^{n} L_{\epsilon n}+\sum_{i^{\prime} \in \bar{S}} \bar{b}_{n_{i^{\prime}}} Y_{i^{\prime}}+\sum_{j^{\prime} \in \bar{S}^{\prime}} \bar{c}_{n_{j^{\prime}}} M_{j^{\prime}}, \\
\theta^{-1} \sigma\left(Y_{n}\right)=\bar{\alpha}^{n} \bar{\beta} Y_{\epsilon n}+\sum_{k \in \bar{S}^{\prime \prime}} \bar{e}_{n_{k}} M_{k}, \\
\theta^{-1} \sigma\left(M_{n}\right)=\epsilon \bar{\alpha}^{n} \bar{\beta}^{2} M_{\epsilon n},
\end{array}\right.
$$

for some $\bar{\alpha}, \bar{\beta} \in \mathbb{C}^{*}, \bar{b}_{n_{i^{\prime}}}, \bar{c}_{n_{j^{\prime}}}$ and $\bar{e}_{n_{k}} \in \mathbb{C}, \epsilon \in\{ \pm 1\}$ with $\epsilon=1$ for $\mu \notin \frac{1}{2} \mathbb{Z}, \bar{S}, \bar{S}^{\prime}, \bar{S}^{\prime \prime} \subseteq \mathbb{Z}$.

Applying $\theta^{-1} \sigma$ to $(n+\mu) Y_{n}=\left[L_{0}, Y_{n}\right]$, we obtain

$$
\begin{aligned}
& (n+\mu)\left(\bar{\alpha}^{n} \bar{\beta} Y_{\epsilon n}+\sum_{k \in \bar{S}^{\prime \prime}} \bar{e}_{n_{k}} M_{k}\right) \\
= & {\left[\epsilon L_{0}+c_{0} M_{0}, \bar{\alpha}^{n} \bar{\beta} Y_{\epsilon n)}+\sum_{k \in \bar{S}^{\prime \prime}} \bar{e}_{n_{k}} M_{k}\right]=(n+\mu) \bar{\alpha}^{n} \bar{\beta} Y_{\epsilon n}+\sum_{k \in \bar{S}^{\prime \prime}} \epsilon \bar{e}_{n_{k}}(k+2 \mu) M_{k}, }
\end{aligned}
$$

which gives

$$
(n+\mu) \sum_{k \in \bar{S}^{\prime \prime}} \bar{e}_{n_{k}} M_{k}=\sum_{k \in \bar{S}^{\prime \prime}} \epsilon \bar{e}_{n_{k}}(k+2 \mu) M_{k}
$$

Comparing the coefficients of $M_{k}$, one has

$$
\bar{e}_{n_{k}}(k+2 \mu-\epsilon(n+\mu))=0, \quad \forall k \in \bar{S}^{\prime \prime} .
$$

Applying $\theta^{-1} \sigma$ to $n L_{n}=\left[L_{0}, L_{n}\right]$, we obtain

$$
\begin{aligned}
& n\left(\epsilon \bar{\alpha}^{n} L_{\epsilon n}+\sum_{i^{\prime} \in \bar{S}} \bar{b}_{n_{i^{\prime}}} Y_{i^{\prime}}+\sum_{j^{\prime} \in \bar{S}^{\prime}} \bar{c}_{n_{j^{\prime}}} M_{j^{\prime}}\right) \\
= & {\left[\epsilon L_{0}+c_{0} M_{0}, \epsilon \bar{\alpha}^{n} L_{\epsilon n}+\sum_{i^{\prime} \in \bar{S}} \bar{b}_{n_{i^{\prime}}} Y_{i^{\prime}}+\sum_{j^{\prime} \in \bar{S}^{\prime}} \bar{c}_{n_{j^{\prime}}} M_{j^{\prime}}\right] } \\
= & \epsilon n \bar{\alpha}^{n} L_{\epsilon n}+\sum_{i^{\prime} \in \bar{S}} \epsilon \bar{b}_{n_{i^{\prime}}}\left(i^{\prime}+\mu\right) Y_{i^{\prime}}+\sum_{j^{\prime} \in \bar{S}^{\prime}} \epsilon \bar{c}_{n_{j^{\prime}}}\left(j^{\prime}+2 \mu\right) M_{j^{\prime}}+\epsilon \bar{\alpha}^{n} c_{0}(\lambda \epsilon n-2 \mu) M_{\epsilon n} .
\end{aligned}
$$

Using $c_{0}(\lambda n-2 \mu)=0$ and $\epsilon=1$ for $\mu \notin \frac{1}{2} \mathbb{Z}$, one can deduce $\epsilon \bar{\alpha}^{n} c_{0}(\lambda \epsilon n-2 \mu)=0$. Using this in (3.31), one has

$$
n\left(\sum_{i^{\prime} \in \bar{S}} \bar{b}_{n_{i^{\prime}}} Y_{i^{\prime}}+\sum_{j^{\prime} \in \bar{S}^{\prime}} \bar{c}_{n_{j^{\prime}}} M_{j^{\prime}}\right)=\sum_{i^{\prime} \in \bar{S}} \epsilon \bar{b}_{n_{i^{\prime}}}\left(i^{\prime}+\mu\right) Y_{i^{\prime}}+\sum_{j^{\prime} \in \bar{S}^{\prime}} \epsilon \bar{c}_{n_{j^{\prime}}}\left(j^{\prime}+2 \mu\right) M_{j^{\prime}}
$$

Comparing the coefficients of $Y_{i^{\prime}}$ and $M_{j^{\prime}}$ respectively, we have

$$
\bar{b}_{n_{i^{\prime}}}\left(i^{\prime}-\epsilon n+\mu\right)=0, \forall i^{\prime} \in \bar{S}, \quad \bar{c}_{n_{j^{\prime}}}\left(j^{\prime}-\epsilon n+2 \mu\right)=0, \quad \forall j^{\prime} \in \bar{S}^{\prime} .
$$


Case $1 \mu \notin \frac{1}{2} \mathbb{Z}$.

Since $\mu \notin \frac{1}{2} \mathbb{Z}$, we have $\epsilon=1$. Thus, by (13.30) and (3.32), we obtain $\bar{b}_{n_{i^{\prime}}}, \bar{c}_{n_{j^{\prime}}}$ and $\bar{e}_{n_{k}}$, are all equal to zero for all $i^{\prime} \in \bar{S}, j^{\prime} \in \bar{S}^{\prime}$ and $k \in \bar{S}^{\prime \prime}$. Using this in (3.29), we obtain

$$
\theta^{-1} \sigma\left(L_{n}\right)=\bar{\alpha}^{n} L_{n}, \quad \theta^{-1} \sigma\left(Y_{n}\right)=\bar{\alpha}^{n} \bar{\beta} Y_{n}, \quad \theta^{-1} \sigma\left(M_{n}\right)=\bar{\alpha}^{n} \bar{\beta}^{2} M_{n}
$$

Let $\varphi_{\bar{\alpha}, \bar{\beta}}$ be the automorphism of $\mathscr{L}$ as that given in Lemma 3.3 (ii). Then $\sigma=\theta \varphi_{\bar{\alpha}, \bar{\beta}}$.

Case $2 \mu=0$.

Since $\mu=0$, we know that $i^{\prime}, j^{\prime}$ and $k$ are all equal to $\epsilon n$ for all $i^{\prime} \in \bar{S}, j^{\prime} \in \bar{S}^{\prime}$ and $k \in \bar{S}^{\prime \prime}$ in (3.30) and (3.32). Using this in (3.29), we obtain

$$
\left\{\begin{array}{l}
\theta^{-1} \sigma\left(L_{n}\right)=\epsilon \bar{\alpha}^{n} L_{\epsilon n}+\bar{b}_{n} Y_{\epsilon n}+\bar{c}_{n} M_{\epsilon n} \\
\theta^{-1} \sigma\left(Y_{n}\right)=\bar{\alpha}^{n} \bar{\beta} Y_{\epsilon n}+\bar{e}_{n} M_{\epsilon n} \\
\theta^{-1} \sigma\left(M_{n}\right)=\epsilon \bar{\alpha}^{n} \bar{\beta}^{2} M_{\epsilon n}
\end{array}\right.
$$

for some $\bar{b}_{n}, \bar{c}_{n}$ and $\bar{e}_{n} \in \mathbb{C}$. Let $\bar{\theta}$ be the automorphism of $\mathscr{L}$ defined by

$$
\bar{\theta}\left(X_{n}\right)=\bar{\alpha}^{n} X_{n} \quad \text { for } X \in\{L, Y, W\}
$$

Then $\bar{\theta}$ is an inner one. Set

$$
\bar{f}(n)=\bar{b}_{n}\left(\bar{\alpha}^{n} \bar{\beta}\right)^{-1}, \quad f(n)=\bar{c}_{n}\left(\epsilon \bar{\alpha}^{n} \bar{\beta}^{2}\right)^{-1}, \quad g(n)=\bar{e}_{n}\left(\epsilon \bar{\alpha}^{n} \bar{\beta}^{2}\right)^{-1}
$$

Define $\varphi_{\epsilon}$ and $\varphi_{1, \bar{\beta}}$ as those given in Lemma 3.3 (i) and (ii) respectively. Then we can write (3.33) as follows

$$
\phi\left(L_{n}\right)=L_{n}+\bar{f}(n) Y_{n}+f(n) M_{n}, \quad \phi\left(Y_{n}\right)=Y_{n}+g(n) M_{n}, \quad \phi\left(M_{n}\right)=M_{n},
$$

where $\phi=\left(\varphi_{\epsilon}\right)^{-1}\left(\varphi_{1, \bar{\beta}}\right)^{-1}(\bar{\theta})^{-1} \theta^{-1} \sigma$.

Subcase $1 \lambda=-2$.

By Lemma 3.2, there exist some $c_{1}, \bar{c}_{1}$ and $b_{1} \in \mathbb{C}$ such that

$$
\begin{aligned}
& \phi\left(L_{n}\right)=L_{n}+b_{1} n Y_{n}+\left(\frac{c_{1}}{6}\left(n^{3}-n\right)-\frac{\bar{c}_{1}}{3}\left(n^{3}-4 n\right)+\frac{b_{1}^{2}}{3} n(n-2)(n-1)\right) M_{n}, \\
& \phi\left(Y_{n}\right)=Y_{n}-2 b_{1} n M_{n}, \quad \phi\left(M_{n}\right)=M_{n} .
\end{aligned}
$$

Set $\theta_{1}=\exp \left(-2 b_{1} \operatorname{ad} Y_{0}+\frac{1}{12}\left(c_{1}-8 \bar{c}_{1}-4 b_{1}^{2}\right) \operatorname{ad} M_{0}\right)$. Then

$$
\left(\theta_{1}\right)^{-1} \phi\left(L_{n}\right)=L_{n}+\frac{c_{1}-2 \bar{c}_{1}+2 b_{1}^{2}}{6} n^{3} M_{n}, \quad\left(\theta_{1}\right)^{-1} \phi\left(Y_{n}\right)=Y_{n}, \quad\left(\theta_{1}\right)^{-1} \phi\left(M_{n}\right)=M_{n} .
$$

Set $\alpha_{1}=\frac{1}{6}\left(c_{1}-2 \bar{c}_{1}+2 b_{1}^{2}\right)$ and define $\phi_{\alpha_{1}}$ as in Lemma 3.3 (iii). Then $\left(\phi_{\alpha_{1}}\right)^{-1}\left(\theta_{1}\right)^{-1} \phi=I d$. 
Subcase $2 \lambda=-1$.

By Lemma 3.2, there exist some $c_{2}, \bar{c}_{2}$ and $b_{2} \in \mathbb{C}$ such that

$$
\phi\left(L_{n}\right)=L_{n}+\left(\frac{c_{2}}{2}\left(n^{2}-n\right)-\bar{c}_{2}\left(n^{2}-2 n\right)\right) M_{n}, \quad \phi\left(Y_{n}\right)=Y_{n}+b_{2} n M_{n}, \quad \phi\left(M_{n}\right)=M_{n} .
$$

Define $\psi_{b_{2}}$ that given as in Lemma 3.3 (iv). Then

$$
\begin{aligned}
& \left(\psi_{b_{2}}\right)^{-1} \phi\left(L_{n}\right)=L_{n}+\left(\frac{c_{2}}{2}\left(n^{2}-n\right)-\bar{c}_{2}\left(n^{2}-2 n\right)\right) M_{n} \\
& \left(\psi_{b_{2}}\right)^{-1} \phi\left(Y_{n}\right)=Y_{n}, \quad\left(\psi_{b_{2}}\right)^{-1} \phi\left(M_{n}\right)=M_{n} .
\end{aligned}
$$

Set $\theta_{2}=\exp \left(\frac{1}{2}\left(c_{2}-4 \bar{c}_{2}\right) \operatorname{ad} M_{0}\right)$. Then

$$
\begin{aligned}
& \left(\theta_{2}\right)^{-1}\left(\psi_{b_{2}}\right)^{-1} \phi\left(L_{n}\right)=L_{n}+\frac{c_{2}-2 \bar{c}_{2}}{2} n^{2} M_{n} \\
& \left(\theta_{2}\right)^{-1}\left(\psi_{b_{2}}\right)^{-1} \phi\left(Y_{n}\right)=Y_{n}, \quad\left(\theta_{2}\right)^{-1}\left(\psi_{b_{2}}\right)^{-1} \phi\left(M_{n}\right)=M_{n} .
\end{aligned}
$$

Set $\alpha_{2}=\frac{1}{2}\left(c_{2}-2 \bar{c}_{2}\right)$, let $\phi_{\alpha_{2}}$ be as that given in Lemma 3.3(iii). Then $\left(\phi_{\alpha_{2}}\right)^{-1}\left(\theta_{2}\right)^{-1}\left(\psi_{b_{2}}\right)^{-1} \phi=$ Id.

Subcase $3 \lambda=1$.

By Lemma 3.2, there exist some $e, c_{4}, \bar{c}_{4}$ and $b_{4} \in \mathbb{C}$ such that

$$
\begin{aligned}
& \phi\left(L_{n}\right)=L_{n}+b_{4} n Y_{n}+\left(c_{4} n+\frac{b_{4}^{2}}{2} n(n-1)\right) M_{n} \\
& \phi\left(Y_{n}\right)=Y_{n}+\left(b_{4} n+e\right) M_{n}, \quad \phi\left(M_{n}\right)=M_{n} .
\end{aligned}
$$

Set $\theta_{4}=\exp \left(b_{4} \operatorname{ad} Y_{0}-\frac{1}{2}\left(b_{4}^{2}-2 c_{4}\right) \operatorname{ad} M_{0}\right)$. Then

$$
\left(\theta_{4}\right)^{-1} \phi\left(L_{n}\right)=L_{n}, \quad\left(\theta_{4}\right)^{-1} \phi\left(Y_{n}\right)=Y_{n}+e M_{n}, \quad\left(\theta_{4}\right)^{-1} \phi\left(M_{n}\right)=M_{n} .
$$

Let $\psi_{e}$ be as that given in Lemma 3.3 (iv). Then $\left(\psi_{e}\right)^{-1}\left(\theta_{4}\right)^{-1} \phi=I d$.

Subcase $4 \lambda \notin\{-2,0, \pm 1\}$.

By Lemma 3.2, there exist some $c_{5}, \bar{c}_{5}$ and $b_{5} \in \mathbb{C}$ such that

$$
\begin{aligned}
& \phi\left(L_{n}\right)=L_{n}+b_{5} n Y_{n}+\left(\bar{c}_{5} n+\frac{b_{5}^{2}}{\lambda+1} n(n-1)\right) M_{n} \\
& \phi\left(Y_{n}\right)=Y_{n}+\frac{2 b_{5}}{\lambda+1} n M_{n}, \quad \phi\left(M_{n}\right)=M_{n} .
\end{aligned}
$$

Set $\theta_{5}=\exp \left(2 b_{5}(\lambda+1)^{-1} \operatorname{ad} Y_{0}+\left(\bar{c}_{5}(\lambda+1)-b_{5}^{2}\right)\left(\lambda^{2}+\lambda\right)^{-1} \operatorname{ad} M_{0}\right)$. Then $\left(\theta_{5}\right)^{-1} \phi=I d$. 
Recall that $\phi=\left(\varphi_{\epsilon}\right)^{-1}\left(\varphi_{1, \bar{\beta}}\right)^{-1}(\bar{\theta})^{-1} \theta^{-1} \sigma$. Since $\operatorname{Inn} \mathscr{L}$ is the normal subgroup of Aut $\mathscr{L}$, then there exist $\bar{\theta}_{i} \in \operatorname{Inn} \mathscr{L}(i=1,2, \cdots, 5)$ such that

$$
\sigma= \begin{cases}\bar{\theta}_{1} \varphi_{1, \bar{\beta}} \varphi_{\epsilon} \phi_{\alpha_{1}} & \text { if } \lambda=-2, \\ \bar{\theta}_{2} \varphi_{1, \bar{\beta}} \varphi_{\epsilon} \phi_{\alpha_{2}} \psi_{b_{2}} & \text { if } \lambda=-1, \\ \bar{\theta}_{4} \varphi_{1, \bar{\beta}} \varphi_{\epsilon} \psi_{e} & \text { if } \lambda=1, \\ \bar{\theta}_{5} \varphi_{1, \bar{\beta}} \varphi_{\epsilon} & \text { if } \lambda \notin\{-2,0, \pm 1\} .\end{cases}
$$

Obviously, Inn $\mathscr{L}$ satisfies (3.26). Hence the theorem follows from Lemma 3.2 .

\section{References}

[1] R. Farnsteiner, Derivations and central extensions of finitely generated graded Lie algebra, J. Alg., 118 (1988), 33-45.

[2] M. Henkel, Schrödinger invariance and strongly anisotropic critical systems, J. Stat. Phys., 75 (1994), 1023-1029.

[3] D. $\breve{Z}$. Doković, K. Zhao, Derivations, isomorphisms and second cohomology of generalized Witt algebras, Tran. Amer. Math. Soc., 350 (1998), 643-664.

[4] Y. Su, Derivations and structure of the Lie algebras of Xu type, Manuscripta Math., 105 (2001), 483-500.

[5] Y. Su, Derivations of generalized Weyl algebras, Science in China A, 46 (2003), 346-354.

[6] G. Song, Y. Su, Derivations and 2-cocycles of contact Lie algebras related to locally-finite derivations, Comm. Alg., 32 (2004), 4613-4631.

[7] M. Henkel, J. Unterberger, Schrödinger invariance and spacetime symmetries, Nucl. Phys. $B, 660$ (2003), 407-435.

[8] M. Henkel, J. Unterberger, Supersymmetric extensions of Schrödinger invariance, Nucl. Phys. B, 746 (2003), 155-201.

[9] C. Roger, J. Unterberger, The Schrödinger-Virasoro Lie group and algebra: representation theory and cohomological study, Ann. Henri Poincaré, 7 (2006), 1477-1529.

[10] J. Unterberger, On vertex algebra representations of the Schrödinger-Virasoro Lie algebra, Nucl. Phys. B, 823 (3) (2009), 320-371.

[11] J. Han, J. Li, Y. Su, Lie bialgebra structures on the Schrödinger-Virasoro Lie algebra, J. Math Phys., 50 (8) (2009): 083504.

[12] J. Li, Y. Su, Representations of the Schrödinger-Virasoro algebras, J. Math Phys., 49 (5) (2008): 053512.

[13] J. Li, Y. Su, The derivation algebra and automorphism group of the twisted SchrödingerVirasoro algebra, arXiv:0801.2207. 
[14] X. Zhang, X. Hu, Derivations of the deformation Schrödinger-Virasoro algebras, J. Xuzhou norm. Univ., 27 (1) (2009): 25-29 (in Chinese).

[15] S. Tan, X. Zhang, Automorphisms and Verma modules for generalized Schrödinger-Virasoro algebras, J. Alg., 322 (2009), 1379-1394.

[16] X. Zhang, S. Tan, H. Lian, Whittaker modules for the Schrödinger-Witt algebra, J. Math. Phys., 51 (8) (2010): 083524.

[17] S. Gao, C. Jiang, Y. Pei, Structure of the extended Schrödinger-Virasoro Lie algebra $\widetilde{\mathfrak{s v},}$ Alg. Colloq., 16 (4) (2009): 549-566.

[18] R. Shen, C. Jiang, The derivation algebra and the automorphism group of the twisted Heisenberg-Virasoro algebra, Comm. Alg., 34 (2006), 2547-2558. 